TITLE:

\title{
The neuroprotective effect of erythropoietin on spinal motor neurons after nerve root avulsion injury in rats.
}

\section{$\operatorname{AUTHOR}(\mathrm{S}):$}

Noguchi, Takashi; Ohta, Souichi; Kakinoki, Ryosuke; Ikeguchi, Ryosuke; Kaizawa, Yukitoshi; Oda, Hiroki; Matsuda, Shuichi

\section{CITATION:}

Noguchi, Takashi ... [et al]. The neuroprotective effect of erythropoietin on spinal motor neurons after nerve root avulsion injury in rats.. Restorative neurology and neuroscience 2015, 33(4): 461-470

\section{ISSUE DATE:}

2015-08-19

URL:

http://hdl.handle.net/2433/202120

\section{RIGHT:}

This PDF file is the manuscript version of this article.; この論文は出版社 版でありません。引用の際には出版社版をご確認ご利用ください。; This is not the published version. Please cite only the published version. 


\section{The neuroprotective effect of erythropoietin on spinal motor}

\section{neurons after nerve root avulsion injury in rats}

Takashi Noguchi, MD., PhD.1, Souichi Ohta, MD., PhD.1,CA, Ryosuke Kakinoki, MD.,

PhD. ${ }^{2}$, Ryosuke Ikeguchi, MD, PhD ${ }^{1}$, Yukitoshi Kaizawa, MD. ${ }^{1}$, Hiroki Oda, MD ${ }^{1}$, Shuichi

Matsuda, MD., PhD. ${ }^{1}$

1; Department of Orthopaedic Surgery, Graduate School of Medicine, Kyoto University

2; Department of Orthopaedic Surgery, Faculty of Medicine, Kindai University

CA; Corresponding Author

1; Department of Orthopaedic Surgery, Graduate School of Medicine, Kyoto University,

54 Shogoin Kawahara-cho, Sakyo-ku, Kyoto City, Kyoto 606-8507, Japan

2; Department of Orthopaedic Surgery, Faculty of Medicine, Kindai University, 377-2

Oono-higashi, Osaka-sayama, Osaka, 589-8511, JAPAN 


\section{Abstract}

Purpose: The rapid death of many spinal motor neurons after nerve root avulsion injury results in limited functional recovery following replantation surgery of avulsed nerves into the spinal cord. Therefore, we investigated the neuroprotective effect of erythropoietin (EPO) on motor neurons after nerve root avulsion injury using a rat model. Methods: After C6 nerve root avulsion injury, EPO (2680 U/kg) was injected subcutaneously once a day for 3 consecutive days with various starting time points. At 28 and 56 days after injury, histological and immunohistological investigations were performed. Results: EPO-treated rats showed a significant increase in the number of surviving motor neurons at day 28 when the initial dose was started within $96 \mathrm{~h}$ after injury. In EPO-treated rats, superoxide formation in the motor neurons and proliferation of microglia were markedly suppressed in the acute phase. GAP-43-positive surviving motor neurons were significantly increased in EPO-treated rats at day 28. However, at 56 days after surgery, EPO-treated rats showed a much greater decrease of surviving motor neurons compared with those at day 28. Conclusion: The neuroprotective effect of EPO is not long lasting, but may prolong the time before replantation surgery.

\section{Keywords:}

Nerve root avulsion injury, Oxidative stress, Rat

Motor neurons, Erythropoietin 


\section{Introduction}

Brachial plexus injury, especially nerve root avulsion injury, is one of the most difficult nerve injuries to treat. As root avulsion involves the disruption of the connection between the spinal cord and peripheral nerve, surgical repair has been considered impossible. Therefore, the current treatment option for nerve root avulsion injury includes nerve transfer, muscle graft or transfer, tendon transfer, or arthrodesis; however, functional recovery in the injured upper extremity is limited and insufficient (Atlan et al., 2012; Chuang, 2009; Doi et al., 1993). Replantation surgery of the avulsed nerve root into the spinal cord was reported in the 1990s. Although this surgery still has a number of unsolved shortcomings, limited functional recovery of the shoulder and elbow joints was seen in some cases (Carlstedt et al., 2000; Carlstedt et al., 2004). One of the important factors for obtaining good functional outcomes following this surgery appears to be the survival of motor neurons within the pertinent spinal cord segment (Carlstedt et al., 2000). After nerve root avulsion injury, the number of motor neurons in the affected spinal cord segment decreases rapidly and is reduced by more than half at 4 weeks (Wu, 1993; Yuan et al., 2009). Therefore, inhibition of motor neuron death may be a therapeutic strategy for nerve root avulsion injury (Havton et al., 2009). The hematopoietic hormone erythropoietin (EPO) shows neuroprotective effects in acute cerebral and spinal cord injuries (Matis et al., 2009; Sakanaka et al., 1998; Velly et al., 2010; Boran et al., 2005). The neuroprotective mechanisms of EPO include antagonizing the cytotoxic effect of glutamate, enhancing the expression of antioxidant enzymes, reducing nitric oxide-mediated formation of free radicals, and influencing 
neurotransmitter release (Buemi et al., 2003). Although nerve root avulsion injury involves rupture of the ventral and dorsal roots, many motor neurons in the ventral horn of the spinal cord die within 4 weeks after injury. The mechanisms of this motor neuron death are still unclear; however, nitric oxide and superoxide have been reported to contribute to motor neuron death (Estevez et al., 1998). We hypothesized that EPO has an inhibitory effect on motor neuron death after nerve root avulsion injury and investigated its effect using rat nerve root avulsion models.

\section{Materials and Methods}

\section{Animals}

Eighty-four 9-week-old male adult Sprague-Dawley rats (Nippon SLC Inc., Hamamatsu, Japan) weighing approximately 280 g (270-300 g) were used. All animals were housed in a room with a daily 14-h light/10-h dark cycle with free access to food and water. Before surgery, rats were anesthetized intraperitoneally with sodium pentobarbital $(40 \mathrm{mg} / \mathrm{kg})$. All animal procedures were performed according to the Guidelines of the Animal Research Committee, Graduate School of Medicine, Kyoto University.

\section{Surgical procedure for C6 nerve root avulsion injury}

Surgery was performed as previously described (Noguchi et al., 2013). In brief, a midline skin 
incision was made at the posterior side of the neck. The left paravertebral muscles of the cervical spine were unilaterally dissected from the spinous processes, laminae, and anterior articular processes (lateral mass) at the level of C4 to Th2. The transverse process and lateral third of the C5 and C6 lateral mass were removed gently using a surgical bur. Then, the C6 nerve root was exposed and pulled gently using Adson forceps with no teeth. Successful nerve root avulsion was confirmed by visualization of the bifurcated stump of the avulsed nerve root and leakage of spinal fluid from the vertebral foramen.

\section{EPO injection}

Commercially available human recombinant EPO (Epoetin alpha) (Espo ${ }^{\circledR}$; Kirin-Hakko, Tokyo, Japan) was used, and $2680 \mathrm{U} / \mathrm{kg}$ was subcutaneously injected into the lower back once a day for 3 consecutive days as previously reported (Sekiguchi et al., 2003). The dose of rhEPO was established based on the bioavailability and clearance rates of rhEPO (Bleuel et al., 1996).

\section{Experiment 1: effect of EPO 28 days after injury}

Forty rats were randomly divided into the following eight groups according to the time of first EPO administration after surgery ( $\mathrm{n}=5$ each): control (saline administration), immediate, 6 h, 24 h, 48 h, $96 \mathrm{~h}, 150 \mathrm{~h}$, and $300 \mathrm{~h}$. At 28 days after surgery, rats in all groups were sacrificed for histological investigation. 


\section{Experiment 2: effect of EPO 56 days after injury}

Twelve other rats were randomly divided into the following two groups ( $\mathrm{n}=6$ each): control (saline administration) and EPO-treated (immediately after surgery). At 56 days after surgery, all rats were sacrificed for histological investigation.

\section{Tissue preparation}

The rats were deeply anesthetized with a lethal dose of sodium pentobarbital and transcardially perfused with $200 \mathrm{~mL}$ phosphate-buffered saline (PBS, $\mathrm{pH}$ 7.4) followed by $350 \mathrm{~mL} 4 \%$ (w/v) paraformaldehyde (PFA) in $0.1 \mathrm{M}$ phosphate buffer (PB, $\mathrm{pH}$ 7.4). Cervical spinal cord segments (5 $\mathrm{mm}$ in length) were removed and post-fixed overnight in perfusion fixative at $4^{\circ} \mathrm{C}$ and cryoprotected for $24 \mathrm{~h}$ in $20 \%(\mathrm{w} / \mathrm{v})$ sucrose in $\mathrm{PB}\left(4^{\circ} \mathrm{C}\right)$. Then, C6 spinal segments were cut in a cryostat into 40- $\mu$ m-thick serial transverse sections. The C6 spinal segment was defined as the area between the lower edge of the C5 dorsal rootlets and the upper edge of the C7 dorsal rootlets.

\section{Motor neuron counting}

For motor neuron counting, all sections were stained with $0.5 \%(\mathrm{w} / \mathrm{v})$ cresyl violet (Nissl stain). The number of surviving motor neurons on the lesioned side of the spinal cord was counted in all sections and compared with the number on the unlesioned side according to our previous report (Noguchi et al., 
2013). Large multipolar cells with abundant cytoplasm in the ventral horn were counted as viable

motoneurons. To avoid double counting, the number of surviving motor neurons was corrected with Abercrombie's formula (Clarke et al., 1995). The absence of cell loss was expressed as $100 \%$ of the contralateral control value. Cell counting for all serial sections was performed by two observers who were blinded to the groups.

\section{Experiment 3: detection of superoxide formation in injured motor neurons}

Superoxide formation in injured motor neurons was detected using hydroethidine (HEt; Invitrogen,

Carlsbad, CA, USA) (Kim et al., 2010). HEt is oxidized to fluorescent ethidium by superoxide radicals and can therefore serve as a marker for superoxide production (Bindokas et al., 1996; Murakami et al., 1998). Six rats were randomly divided into the following two groups: EPO-treated group (2680 U/kg EPO was subcutaneously injected only once immediately after surgery; $n=3$ ) and control group ( $1 \mathrm{~mL}$ saline was injected immediately after the surgery; $\mathrm{n}=3$ ). At $24 \mathrm{~h}$ after surgery, the rats were intravenously injected with $1 \mathrm{~mL}$ HEt $(1 \mathrm{mg} / \mathrm{mL})$ solution. After $2 \mathrm{~h}$, the animals were deeply anesthetized with a lethal dose of sodium pentobarbital and then transcardially perfused with $0.1 \mathrm{M} \mathrm{PB}$ at $4^{\circ} \mathrm{C}$. Subsequently, the cervical spinal cord segments were removed and embedded in OCT compound followed by quick freezing in liquid nitrogen. Then, the central region of the C6 spinal segments (500- $\mu \mathrm{m}$ length) was cut in a cryostat into $10-\mu \mathrm{m}$-thick serial transverse sections. The sections were fixed with 2\% (w/v) PFA for 1 min, washed twice with PBS, and mounted in 50\% (v/v) 
glycerol. Fluorescent images were obtained using a fixed-power laser with a confocal laser fluorescent microscope (NICON EZ-C1, Tokyo, Japan). The number of oxidized-Het-positive cells was counted. Cell counting for all serial sections was performed by two observers who were blinded to the groups. Results were expressed as the mean number of oxidized-HEt-positive cells per section.

Experiment 4: immunohistochemical analysis for growth associated protein 43 (GAP-43) and Iba-1

Twenty rats were used and randomly divided into the following two groups ( $\mathrm{n}=10$ each): control (saline) and EPO-treated (immediately after surgery). Ten rats were sacrificed on day 3 for immunostaining for Iba1 to detect activated microglia (control; n=5, EPO-treated; n=5). Another 10 rats were sacrificed on day 28 for GAP-43 to detect the regenerative capacity of the injured motor neurons (control; n=5, EPO-treated; $n=5$ ) (Yuan et al., 2009). After transcardiac perfusion with PFA, removed C6 spinal segments were cut in a cryostat into 40 - $\mu$ m-thick serial transverse sections. The primary antibodies used for immunohistochemistry were rabbit polyclonal anti-Iba-1 (1:2000; Wako, Osaka, Japan) and rabbit polyclonal anti-GAP-43 (1:200; Chemicon, Temecula, CA, USA). Staining was performed according to the manufacturer's protocol (Vector ABC Elite kit; Vector, Burlingame, CA, USA). The sections were pretreated with $0.3 \%$ (v/v) $\mathrm{H}_{2} \mathrm{O}_{2}$ in PBS and preincubated with $2 \%$ (v/v) normal goat serum in $0.2 \%(\mathrm{v} / \mathrm{v})$ TritonX-100 in PBS at room temperature. Then, the sections were incubated overnight with the primary antibodies at $4^{\circ} \mathrm{C}$. Secondary biotinylated anti-rabbit antibody (1:200; Vector) was applied for $1 \mathrm{~h}$, followed by incubation with the avidin-biotin-peroxidase 
complex reagent at room temperature for $30 \mathrm{~min}$. The immunoreaction was visualized with diaminobenzidine, and sections were counterstained with an eight-fold dilution of Mayer's hematoxylin solution (Wako, Osaka, Japan). The brown cells with nuclei in the lesioned ventral horn were considered immuno-positive. The number of immune-positive cells was counted using automated counting technique with Image J software (NIH, Bethesda, MD, USA). In brief, after the captured images were converted to grayscale images, the threshold was adjusted manually to separate the particles of interest from background. Then, the number of particles was counted. Cell counting for all serial sections was performed by two observers who were blinded to groups. Results were expressed as the mean number of immuno-positive cells per section.

\section{Experiment 5: erythropoietin receptor (EPOR) expression in avulsed motor neurons}

Six rats were used and randomly sacrificed on day 3, 7, and 14 after avulsion injury for EPOR (n=2 at each time point). No injection was performed after injury. After transcardiac perfusion with PFA,

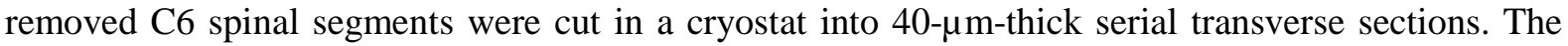
primary antibody used was rabbit polyclonal anti-EPOR (M-20; 1:200; Santa Cruz, CA, USA). Application of secondary antibody, visualization with diaminobenzidine, counterstaining, and cell counting were performed as mentioned above. Results were expressed as the mean number of immuno-positive cells per section. 


\section{Statistical analysis}

All data were expressed as the mean \pm standard error. The data from Experiment 1, 2, 4 (Iba-1), and 5 were compared using one-way analysis of variance followed by Dunnett’s test or Tukey’s test, respectively (JMP 8, SAS Institute, Cary, NC, USA). The data from Experiment 3, and 4 (GAP-43) were compared using the unpaired t-test after normal distribution was confirmed. Statistical significance was set at $\mathrm{p}<0.05$.

\section{Results}

\section{Experiment 1: effects of EPO 28 days after injury}

At 28 days after surgery, the number of surviving motor neurons on the lesioned side decreased in rats in both groups (Fig. 1). However, EPO-treated rats showed significantly more improvement of surviving motor neurons compared with control rats when the initial dose was started within $96 \mathrm{~h}$ after surgery (control: $47.9 \% \pm 4.0$, immediately: $74.2 \% \pm 5.8,6$ h: $79 \% \pm 3.3,24$ h: $77.6 \% \pm 8.4$, 48 h: 75.5\% \pm 4.5, 96 h: 73.9\% \pm 7.6, 150 h: 53.1\% \pm 3.5, 300 h: 51.7\% $\pm 0.8, \mathrm{p}<0.01$ ) (Fig. 2).

\section{Experiment 2: effects of EPO 56 days after injury}

At 56 days after surgery, rats in each group showed a much greater decrease in the percentage of surviving motor neurons on the lesioned side compared with those 28 days after surgery (EPO treated: $74.2 \% \pm 5.8$ at day $28,45.6 \% \pm 4.4$ at day 56 , control: $47.9 \% \pm 4.0$ at day $28,31.8 \% \pm 4.0$ at day 56 , 
$\mathrm{p}<0.01$ ) (Fig. 3). The ratio of surviving motor neurons in rats in the EPO-treated group was significantly higher than that in rats in the control group $(\mathrm{p}<0.01)$.

\section{Experiment 3: superoxide formation in injured motor neurons}

Superoxide in injured motor neurons oxidizes HEt to the red fluorescent dye ethidium. Thus, red motor neurons have an increased formation of intracellular superoxide. In EPO-treated rats, the number of oxidized-HEt-positive cells on the lesion side of the ventral horn significantly decreased compared with control rats $24 \mathrm{~h}$ after surgery (EPO treated: $1.7 \pm 0.7$, control: $12.3 \pm 2.2$, p $<0.05$ ) (Fig .4)

\section{Experiment 4: immunohistochemical analysis for GAP-43 and Iba-1}

\section{Microglial reaction in the lesioned ventral horn}

At day 3 after surgery, rats in both groups showed increased numbers of Iba-1-immunopositive cells on the lesioned side of the ventral horn compared with the unlesioned side of the ventral horn (unlesioned: $14.7 \pm 2.0$, EPO treated: $80.9 \pm 8.9$, control: $138.3 \pm 12.1, \mathrm{p}<0.01$ ) (Fig. 5D). However, the number of Iba-1-immunopositive cells was significantly lower in EPO-treated rats compared with control rats $(\mathrm{p}<0.01)$. Almost all microglia in the unlesioned side showed a ramified form (inset of Fig. 5A). In contrast, many microglia on the lesioned side showed a morphological change to an amoeboid form (inset of Fig.5B and C). 


\section{The regenerative capacity of injured motor neurons}

At day 28 after surgery, the number of GAP-43-immunopositive cells on the lesioned side of the ventral horn was significantly higher in EPO-treated rats compared with control rats (EPO: $3.8 \pm 0.6$, control: $0.8 \pm 0.2, \mathrm{p}<0.01$ ). The percentage of surviving motor neurons immuno-positive for GAP-43 on the lesioned side was $75.7 \pm 3.7 \%$ in EPO-treated rats and $29.1 \pm 9.8 \%$ in control rats $(\mathrm{p}<0.01)$ (Fig. 6).

\section{Experiment 5: EPOR expression in avulsed motor neurons}

EPOR expression was observed in the cell bodies of all motor neurons on both sides of the ventral horn. No significant difference was found in EPOR expression in motor neurons on the lesioned side among the assessed time points (unlesioned side: $9.6 \pm 0.5$, day 3: $9.5 \pm 0.5$, day 7: $8.9 \pm 0.5$, day 14: $7.9 \pm 0.5)$ (Fig. 7).

\section{Discussion}

The present study showed that 1) subcutaneous injection of EPO once a day for 3 days significantly increased the number of surviving motor neurons 28 days after nerve root avulsion injury even when the first administration was delayed until $96 \mathrm{~h}$ after injury, 2) in EPO-treated rats, superoxide formation in the motor neurons and proliferation of microglia were markedly suppressed in the acute 
phase, 3) GAP-43-positive surviving motor neurons were significantly increased in EPO-treated rats at day 28, and 4) at 56 days after injury, a much greater decrease in the percentage of surviving motor neurons on the lesioned side compared with those 28 days after surgery. However, EPO-treated rats still showed a significant increase in surviving motor neurons compared with control rats.

Traumatic injury to the central nervous system results in immediate mechanical damage followed by prolonged secondary injury to neural tissues (Tator et al., 1991). Unlike spinal cord contusion injury, the primary mechanical damage caused by nerve root avulsion is usually minor in the spinal cord (Noguchi et al., 2013). However, many motor neurons in the ventral horn die within 4 weeks after nerve root avulsion injury. A variety of cytotoxic mechanisms evolving from primary mechanical damage are induced, such as so-called secondary spinal cord injury, which progresses irreversibly (Borgens et al., 2012). This delayed injury mechanism is one of the targets for therapeutic intervention to improve treatment for nerve root avulsion injury.

In our study, the subcutaneous injection of EPO significantly increased the number of surviving motor neurons 4 weeks after injury. After axonal injury, neuron-derived nitric oxide stimulates EPO production by neighboring Schwann cells (Keswani et al., 2004). Then, this Schwann cell-derived EPO results in activation of an "axonoprotective" pathway via EPOR ligation on neurons. After root avulsion injury, endogenous EPO produced by Schwann cells in the remaining short ventral rootlets is exhausted shortly after injury. Therefore, systemic administration of EPO may counteract the insufficient production of endogenous EPO. 
In our previous rat study, although the ventral roots were disconnected within the subarachnoid space in both an intradural rhizotomy group and a nerve root avulsion group, the root avulsion group showed a significant decrease in motor neurons compared with the rhizotomy group (Noguchi et al., 2013). Only the root avulsion group showed intraspinal bleeding along motor neuron axons $12 \mathrm{~h}$ after injury. Therefore, we inferred that the traction force to the nerve root causes severe injury to motor neurons and their axons and affects the difference in motor neuron survival between the two groups. However, motor neuron death after root avulsion injury is likely caused by not only the mechanical insult but also other neurotoxic mechanisms (Carlstedt, 2008). The intraspinal bleeding along motor neuron axons after nerve root avulsion injury includes iron, which is an important initiator of free radical oxidation (Halliwell, 1992). Free radicals are neurotoxic substances in the central nervous system. In the present study, EPO-treated rats showed a marked decrease in HEt staining compared with control rats $24 \mathrm{~h}$ after injury. One of the neuroprotective mechanisms of EPO after nerve root avulsion injury is likely suppression of free radical formation.

In the normal central nervous system, ramified resting microglia constantly monitor the surrounding microenvironment (Nimmerjahn et al., 2005). Injury to nervous tissue induces activation of resting microglia via various “danger signals” from neighboring damaged neuronal cells (Loane et al., 2010). Then, depending on the degree of activation, microglia release neurotrophic and anti-inflammatory factors for neuron survival or neurotoxic factors such as superoxide, nitric oxide, and tumor necrosis factor- $\alpha$ (Block et al., 2007). In the present study, EPO-treated rats showed a significant decrease in 
activated microglia at 3 days after nerve root avulsion injury. The suppression of intraneuronal free radical formation by EPO release less “danger signals” from neurons and results in an indirect inhibitory effect on microglial activation. Moreover, with the antioxidant action of EPO, the microenvironment produced by already activated microglia may be modulated to a less favorable one for the development of free radicals (Wenker et al., 2013).

After spinal contusion injury, administration of free radical scavengers as early as possible shows a neuroprotective effect by inhibiting the secondary spinal cord injury process (Ohta et al., 2011). However, our study showed that EPO significantly improved motor neuron survival even when the first administration was delayed until 4 days after nerve root avulsion injury. Compared with spinal cord contusion injury, parenchymal mechanical damage is minor in nerve root avulsion injury (Noguchi et al., 2013). Therefore, nerve root avulsion causes less neurotoxic events induced by free radical formation compared with spinal cord contusion injury. Thus, the window of opportunity for administration of EPO after nerve root avulsion injury is longer than that after spinal contusion injury. Although EPORs were detected in motor neurons on the lesioned side of the ventral horn, administration of EPO did not provide a neuroprotective effect when the initial dose was started 1 or 2 weeks after injury. This may be because an irreversible cascade of neuronal cell death may have already started.

The up-regulation of GAP-43 in motor neurons after root avulsion injury has been reported to correlate with motor neuronal regeneration (Yuan et al., 2009). Yuan et al. showed that GAP-43 was 
expressed within 2 weeks after injury and returned to minimal levels at 4 weeks. Therefore, the preferred timing of replantation surgery was suggested to be within 2 weeks after injury. In the present study, the administration of EPO induced a significant increase in the number of GAP-43 immuno-positive surviving motor neurons at 4 weeks after injury. EPO also prolonged the regeneration capacity of avulsed motor neurons.

Delayed replantation surgery was reported to show a significant decrease in not only survival, but also regeneration of avulsed spinal motor neurons compared with replantation surgery immediately after injury. If replantation surgery of the avulsed nerve roots is performed as early as possible, better functional recovery would be expected. However, in clinical cases, the diagnosis and treatment for brachial plexus avulsion injuries are usually delayed for various reasons such as coexisting life-threatening injuries. Even if a patient is able to consult a specialist early after injury, at least several weeks may be required to receive a detailed diagnosis and subsequent surgery. If medication becomes available that will prevent neuronal loss and prolong the regeneration capacity of motor neurons after avulsion injury, the time before replantation surgery can be extended.

In the present study, the dosage of EPO was determined according to bioavailability and clearance rates (Sekiguchi et al., 2003). EPO exerts a neuroprotective effect at a higher dose compared with the erythropoietic dose. Therefore, when using EPO as a neuroprotective agent, undesirable erythrocytosis and a prothrombotic state may result. However, this may not be a substantial problem for short-term administration of EPO (Kwon et al., 2005). The present study showed a marked 
decrease in preserved motor neurons 56 days after surgery in EPO-treated rats compared with 28 days after surgery. This limited neuroprotective effect on motor neurons was provided by 3 days of EPO administration. Although a longer period of EPO administration may provide a greater neuroprotective effect, this shorter administration time would be sufficient to increase the time before replantation surgery can be performed.

\section{Conclusion}

The present study indicates that EPO has a neuroprotective effect on spinal motor neurons after nerve root avulsion injury. EPO-treated rats showed a significant increase in the number of surviving motor neurons at day 28 when the initial dose was started within $96 \mathrm{~h}$ after injury. The neuroprotective effect of EPO after root avulsion injury did not last long, but would be sufficient to increase the time before commencement of replantation surgery. 


\section{References}

Atlan, F., Durand, S., Fox, M., Levy, P., Belkheyar, Z. \& Oberlin, C. (2012). Functional outcome of glenohumeral fusion in brachial plexus palsy: a report of 54 cases. J Hand Surg Am, 37, 683-688.

Chuang, D.C. (2009). Adult brachial plexus reconstruction with the level of injury: review and personal experience. Plast Reconstr Surg, 124, e359-369.

Doi, K., Sakai, K., Ihara, K., Abe, Y., Kawai, S. \& Kurafuji, Y. (1993). Reinnervated free muscle transplantation for extremity reconstruction. Plast Reconstr Surg, 91, 872-883.

Carlstedt, T., Anand, P., Hallin, R., Misra, P.V., Noren, G. \& Seferlis, T. (2000). Spinal nerve root repair and reimplantation of avulsed ventral roots into the spinal cord after brachial plexus injury. $J$ Neurosurg, 93, 237-247.

Carlstedt, T., Anand, P., Htut, M., Misra, P. \& Svensson, M. (2004). Restoration of hand function and so called "breathing arm" after intraspinal repair of C5-T1 brachial plexus avulsion injury. Case report. Neurosurg Focus, 16, E7.

Wu, W. (1993). Expression of nitric-oxide synthase (NOS) in injured CNS neurons as shown by NADPH diaphorase histochemistry. Exp Neurol, 120, 153-159.

Yuan, Q., Hu, B., Su, H., So, K.F., Lin, Z. \& Wu, W. (2009). GAP-43 expression correlates with spinal motoneuron regeneration following root avulsion. J Brachial Plex Peripher Nerve Inj, 4, 18.

Havton, L.A. \& Carlstedt, T. (2009). Repair and rehabilitation of plexus and root avulsions in animal models and patients. Curr Opin Neurol, 22, 570-574. 
Matis, G.K. \& Birbilis, T.A. (2009). Erythropoietin in spinal cord injury. Eur Spine J, 18, 314-323.

Sakanaka, M., Wen, T.C., Matsuda, S., Masuda, S., Morishita, E., Nagao, M. \& Sasaki, R. (1998). In vivo evidence that erythropoietin protects neurons from ischemic damage. Proc Natl Acad Sci U S A, 95, 4635-4640.

Velly, L., Pellegrini, L., Guillet, B., Bruder, N. \& Pisano, P. (2010). Erythropoietin 2nd cerebral protection after acute injuries: a double-edged sword? Pharmacol Ther, 128, 445-459.

Boran, B.O., Colak, A. \& Kutlay, M. (2005). Erythropoietin enhances neurological recovery after experimental spinal cord injury. Restor Neurol Neurosci, 23, 341-345.

Buemi, M., Cavallaro, E., Floccari, F., Sturiale, A., Aloisi, C., Trimarchi, M., Corica, F. \& Frisina, N. (2003). The pleiotropic effects of erythropoietin in the central nervous system. J Neuropathol Exp Neurol, 62, 228-236.

Estevez, A.G., Spear, N., Manuel, S.M., Radi, R., Henderson, C.E., Barbeito, L. \& Beckman, J.S. (1998). Nitric oxide and superoxide contribute to motor neuron apoptosis induced by trophic factor deprivation. J Neurosci, 18, 923-931.

Noguchi, T., Ohta, S., Kakinoki, R., Kaizawa, Y. \& Matsuda, S. (2013). A new cervical nerve root avulsion model using a posterior extra-vertebral approach in rats. J Brachial Plex Peripher Nerve Inj, $8,8$.

Sekiguchi, Y., Kikuchi, S., Myers, R.R. \& Campana, W.M. (2003). ISSLS prize winner: Erythropoietin inhibits spinal neuronal apoptosis and pain following nerve root crush. Spine (Phila Pa 
1976), 28, 2577-2584.

Bleuel, H., Hoffmann, R., Kaufmann, B., Neubert, P., Ochlich, P.P. \& Schaumann, W. (1996). Kinetics of subcutaneous versus intravenous epoetin-beta in dogs, rats and mice. Pharmacology, 52, 329-338.

Clarke, P.G. \& Oppenheim, R.W. (1995). Neuron death in vertebrate development: in vitro methods. Methods Cell Biol, 46, 277-321.

Kim, D., You, B., Jo, E.K., Han, S.K., Simon, M.I. \& Lee, S.J. (2010). NADPH oxidase 2-derived reactive oxygen species in spinal cord microglia contribute to peripheral nerve injury-induced neuropathic pain. Proc Natl Acad Sci U S A, 107, 14851-14856.

Bindokas, V.P., Jordan, J., Lee, C.C. \& Miller, R.J. (1996). Superoxide production in rat hippocampal neurons: selective imaging with hydroethidine. J Neurosci, 16, 1324-1336.

Murakami, K., Kondo, T., Kawase, M., Li, Y., Sato, S., Chen, S.F. \& Chan, P.H. (1998). Mitochondrial susceptibility to oxidative stress exacerbates cerebral infarction that follows permanent focal cerebral ischemia in mutant mice with manganese superoxide dismutase deficiency. $J$ Neurosci, $18,205-213$.

Tator, C.H. \& Fehlings, M.G. (1991). Review of the secondary injury theory of acute spinal cord trauma with emphasis on vascular mechanisms. J Neurosurg, 75, 15-26.

Borgens, R.B. \& Liu-Snyder, P. (2012). Understanding secondary injury. Q Rev Biol, 87, 89-127.

Keswani, S.C., Buldanlioglu, U., Fischer, A., Reed, N., Polley, M., Liang, H., Zhou, C., Jack, C., Leitz, G.J. \& Hoke, A. (2004). A novel endogenous erythropoietin mediated pathway prevents axonal 
degeneration. Ann Neurol, 56, 815-826.

Carlstedt, T. (2008). Root repair review: basic science background and clinical outcome. Restor Neurol Neurosci, 26, 225-241.

Halliwell, B. (1992). Reactive oxygen species and the central nervous system. J Neurochem, 59, 1609-1623.

Nimmerjahn, A., Kirchhoff, F. \& Helmchen, F. (2005). Resting microglial cells are highly dynamic surveillants of brain parenchyma in vivo. Science, 308, 1314-1318.

Loane, D.J. \& Byrnes, K.R. (2010). Role of microglia in neurotrauma. Neurotherapeutics, 7, 366-377.

Block, M.L., Zecca, L. \& Hong, J.S. (2007). Microglia-mediated neurotoxicity: uncovering the molecular mechanisms. Nat Rev Neurosci, 8, 57-69.

Wenker, S.D., Chamorro, M.E., Vittori, D.C. \& Nesse, A.B. (2013). Protective action of erythropoietin on neuronal damage induced by activated microglia. FEBS J, 280, 1630-1642.

Ohta, S., Iwashita, Y., Kakinoki, R., Noguchi, T. \& Nakamura, T. (2011). Effects of continuous intravenous infusion of MCI-186 on functional recovery after spinal cord injury in rats. $J$ Neurotrauma, 28, 289-298.

Kwon, B.K., Fisher, C.G., Dvorak, M.F. \& Tetzlaff, W. (2005). Strategies to promote neural repair and regeneration after spinal cord injury. Spine (Phila Pa 1976), 30, S3-13. 


\section{Figure Legends}

Figure 1.

Representative images of the C6 ventral horn 28 days after surgery. A: unlesioned side. B: lesioned side in EPO-treated rats (first administration was $24 \mathrm{~h}$ after injury). C: lesioned side in control rats. More surviving motor neurons were seen in EPO-treated rats compared with control rats (Nissl staining, Bar: $100 \mu \mathrm{m})$.

Figure 2.

The relationship between timing of the initial administration of EPO after surgery for 3 days and the number of surviving motor neurons $(\mathrm{n}=5$ each). Starting EPO administration within $96 \mathrm{~h}$ of surgery produced a significant increase in the number of surviving motor neurons 28 days after injury $\left({ }^{*} \mathrm{p}<\right.$ 0.01). All data are the mean \pm SE.

Figure 3.

Time course of the number of surviving motor neurons after injury ( $\mathrm{n}=6$ each). The number of surviving motor neurons in both groups decreased with time. Compared with control rats, the EPO-treated rats demonstrated a significantly higher ratio of surviving motor neurons at 28 and 56 days after surgery $(* \mathrm{p}<0.05)$. 
Figure 4.

Detection of superoxide formation using hydroethidine (HEt) in motor neurons in the lesioned ventral horn $24 \mathrm{~h}$ after surgery. A: unlesioned side in control rats. B: lesioned side in EPO-treated rats (only once immediately after surgery. $\mathrm{n}=3)$. C: lesioned side in control rats $(\mathrm{n}=3)$. D: In EPO-treated rats, the number of oxidized-HEt-positive cells on the lesioned side of the ventral horn was significantly decreased compared with those in control rats $24 \mathrm{~h}$ after surgery $\left({ }^{*} \mathrm{p}<0.05\right)($ Bar: $50 \mu \mathrm{m})$.

Figure 5.

Immunohistochemistry for Iba-1 3 days after surgery (control; $n=5$, EPO-treated; $n=5$ ). A: unlesioned side in control rats. B: lesioned side in EPO-treated rats. C: lesioned side in control rats. Almost all microglia in the unlesioned side showed a ramified form (inset of A). In contrast, many microglia on the lesioned side showed a morphological change to an amoeboid form (inset of B and C). Iba-1-immunopositive cells were significantly increased in the ventral horn on the lesioned side compared with the unlesioned side $(* \mathrm{p}<0.01)(\mathrm{D})$. Iba-1-immunopositive cells were significantly reduced in EPO-treated rats compared with control rats $\left({ }^{* *} \mathrm{p}<0.01\right)$ (Bar: $\left.100 \mu \mathrm{m}\right)$.

Figure 6.

Immunohistochemistry for GAP-43 28 days after surgery (control; $n=5$, EPO-treated; $n=5$ ). A: a representative image of the lesioned C6 ventral horn in EPO-treated rats (A) and control rats (B) (Bar: 
$100 \mu \mathrm{m})$. Black Arrows indicate surviving motor neurons immuno-positive for GAP-43 and white arrows indicate surviving motor neurons immuno-negative for GAP-43. The representative enlarged image of surviving motor neurons immune-positive and immune-negative for GAP-43 (C) (Bar: $50 \mu \mathrm{m})$. The ratio of GAP-43-immunopositive cells was significantly increased in EPO-treated rats compared with control rats $(* \mathrm{p}<0.01)(\mathrm{D})$.

Figure 7.

Immunohistochemistry for EPOR at 3, 7, and 14 days after surgery ( $\mathrm{n}=2$ each). Representative images of the unlesioned C6 ventral horn on day 3 (A), lesioned side on day 3 (B), lesioned side on day 7 (C), and lesioned side on day 14 (D) (Bar: $100 \mu \mathrm{m}$ ). No significant difference was found in EPOR expression in motor neurons among the lesioned side at 3, 7, and 14 days after surgery (E). 
Figure 1.

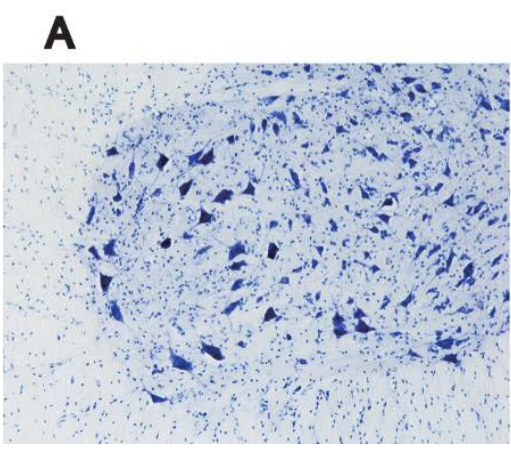

\section{B}

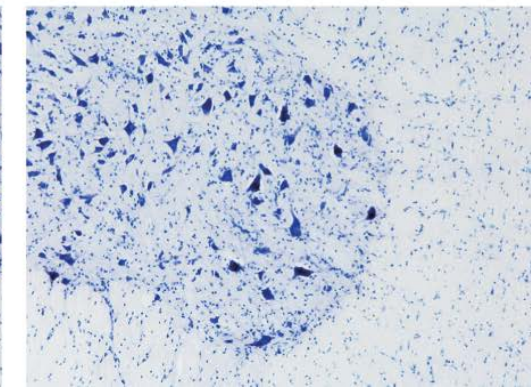

\section{C}

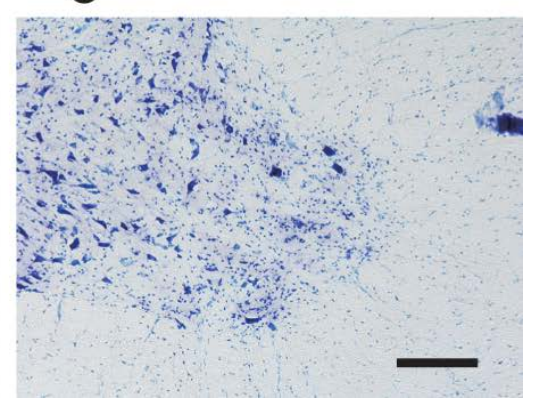

Figure 2.

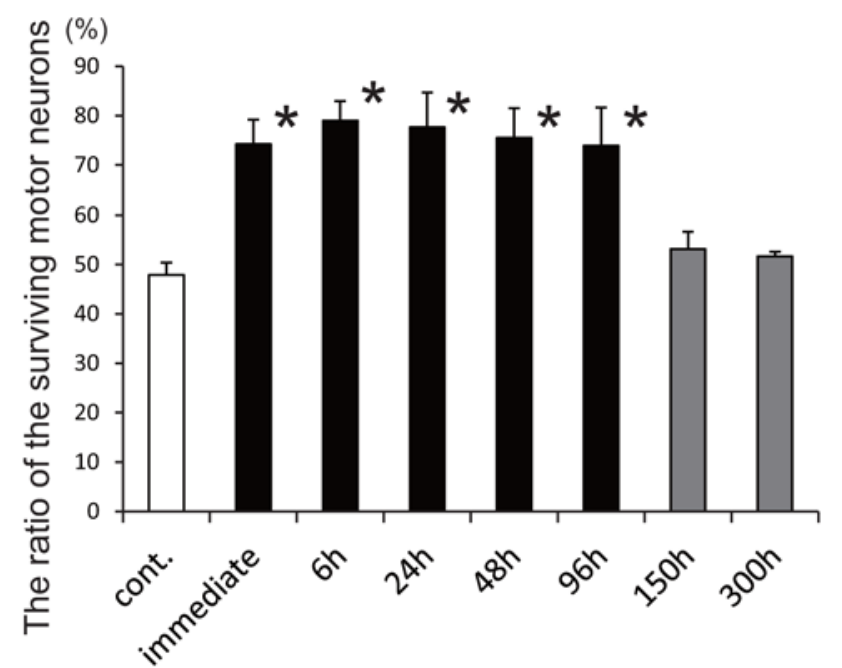

Starting time of EPO administration after injury 
Figure 3.

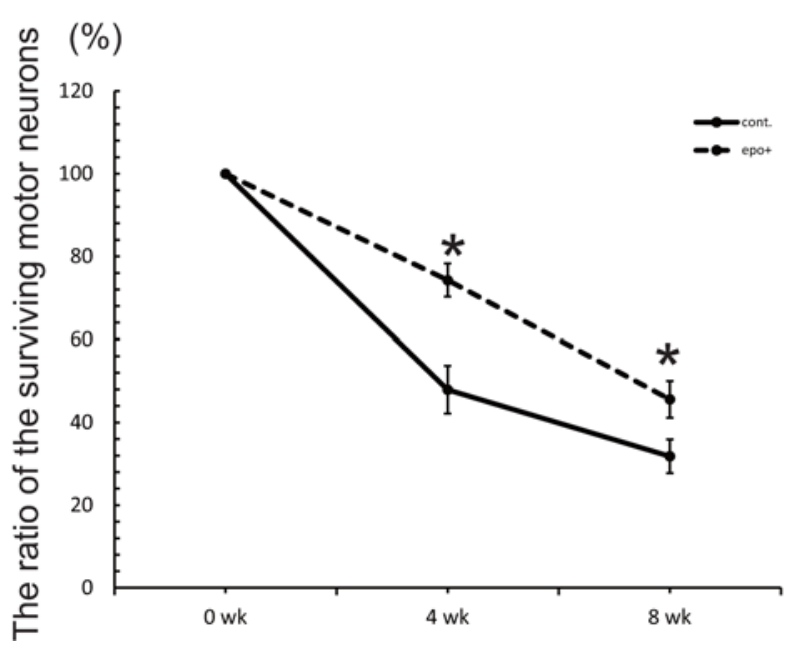

Figure 4.

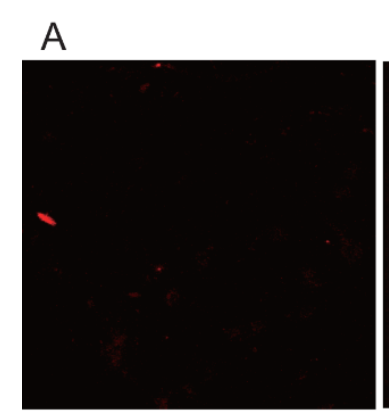

Unlesion

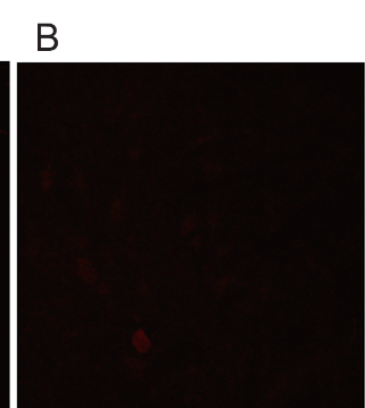

$\mathrm{EPO}(+)$

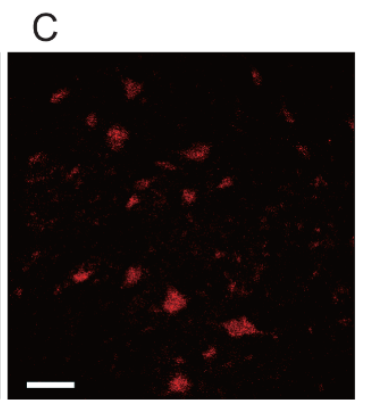

Control

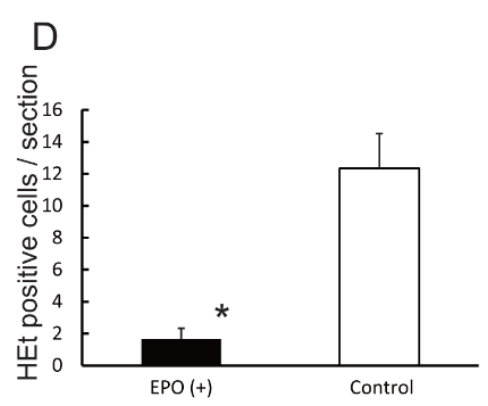


Figure 5.
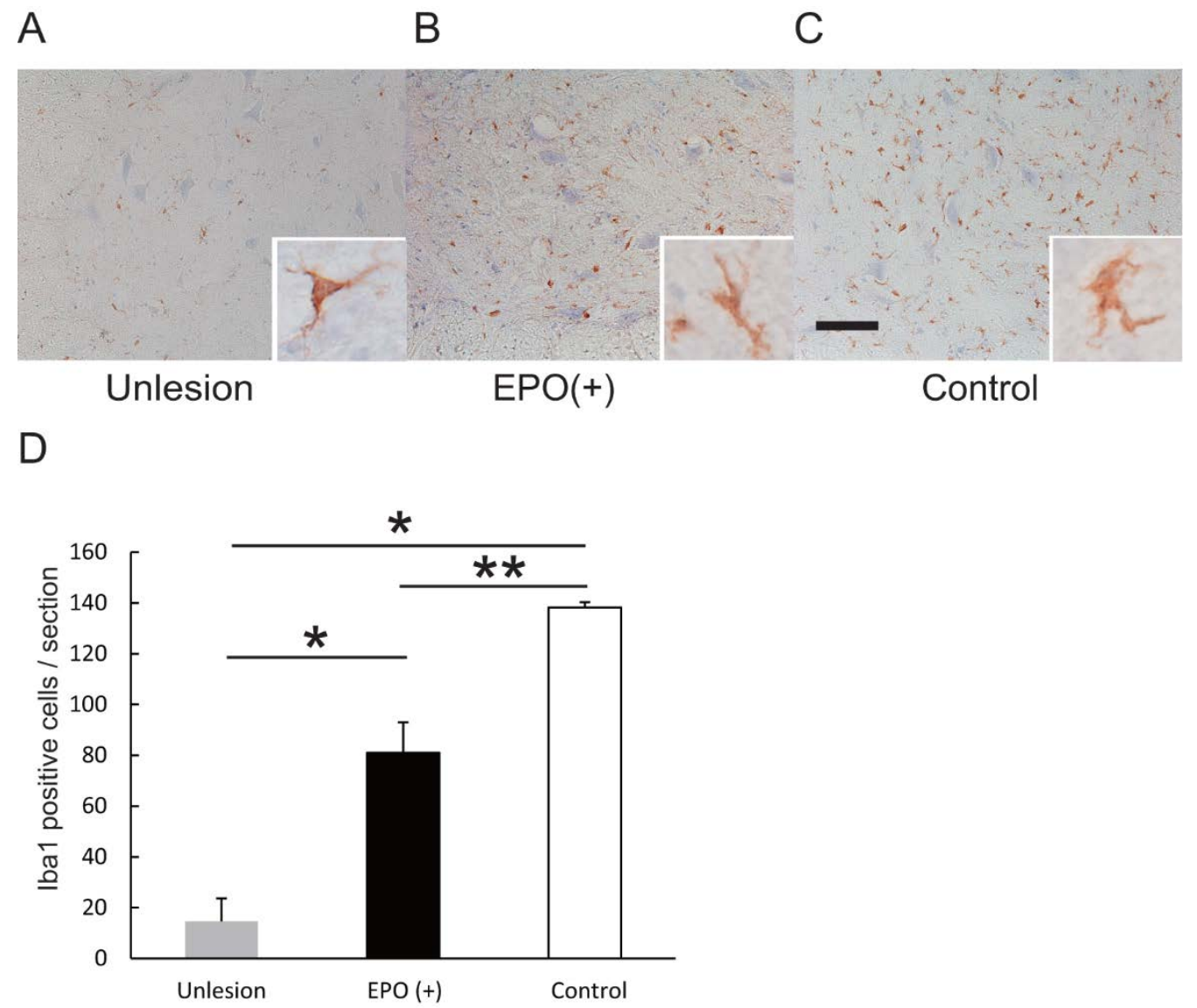

Figure 6.

A

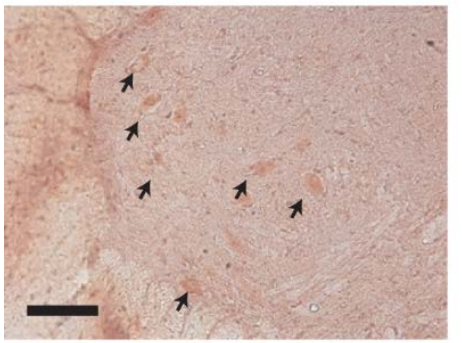

$\mathrm{EPO}(+)$

C

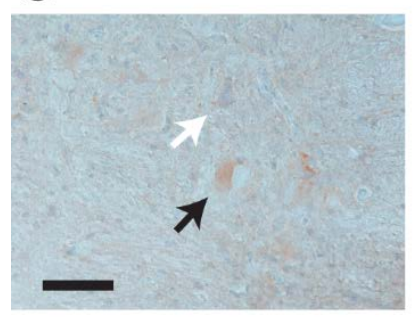

B

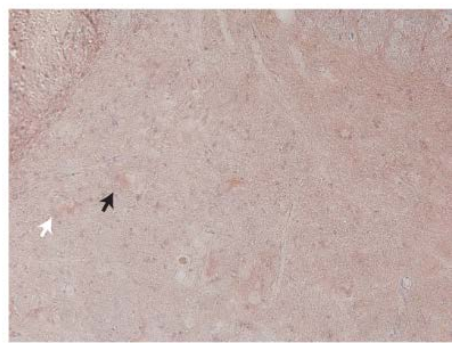

Control

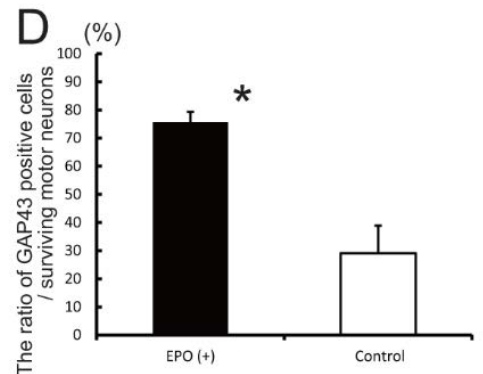


Figure 7.

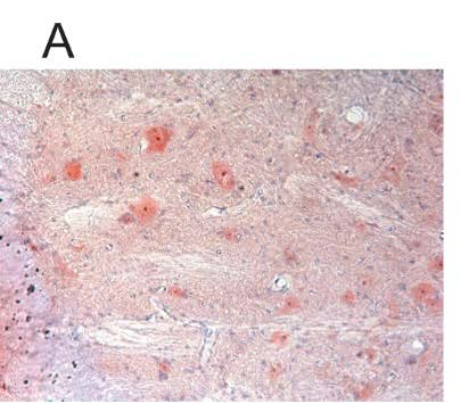

B

C
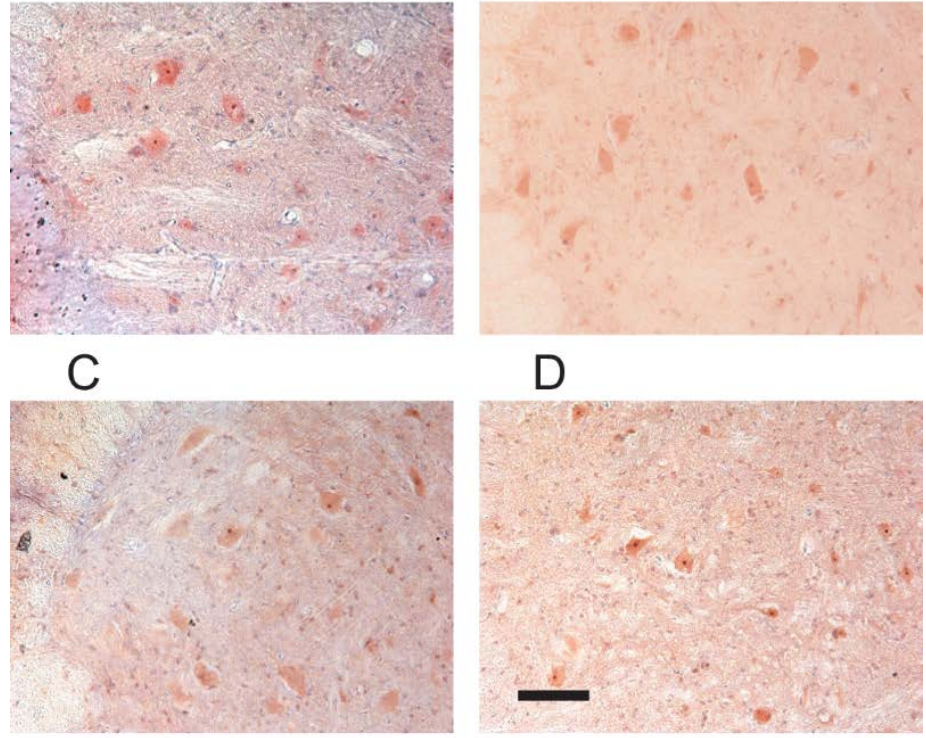

D
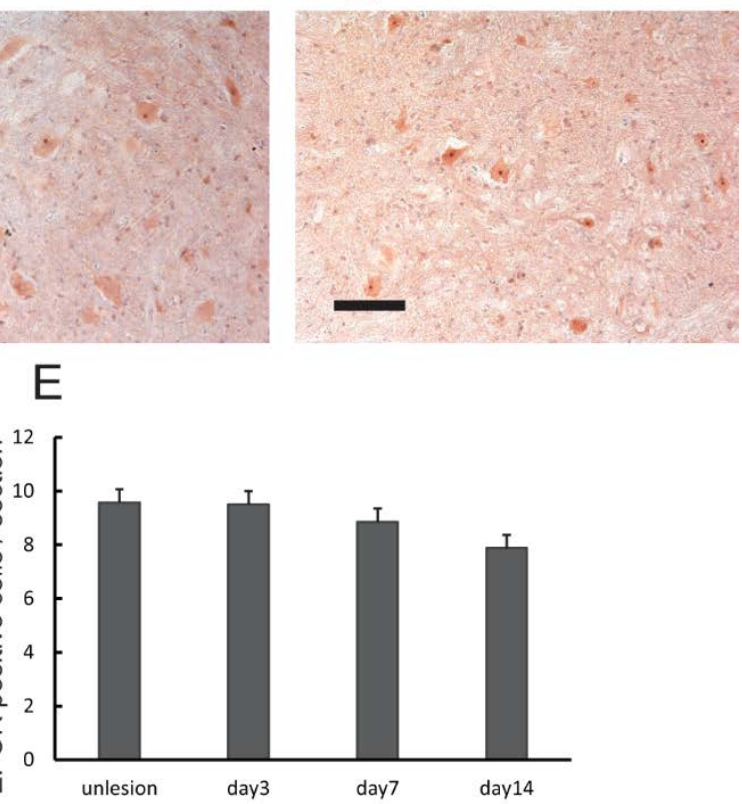